\title{
Phosphorylation Inhibition
}

National Cancer Institute

\section{Source}

National Cancer Institute. Phosphorylation Inhibition. NCI Thesaurus. Code C41594.

Phosphorylation Inhibition involves interference with, or restraint of, the enzymatic creation of a phosphate derivative of an org anic molecule; often by transfer of a phosphate group from ATP via the action of a kinase. 